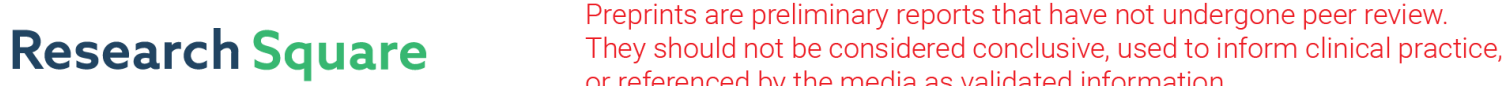 or referenced by the media as validated information. \\ A Stochastic Model for Kala-azar Transmission Dynamics in Libo Kemkem, Ethiopia
}

Sewmehon Shimekaw Alemu ( $\square$ shisewmehon@gmail.com )

University of Gondar

Research

Keywords: Stochastic Model, SIR Model, Infectious Disease, Kala-azar

Posted Date: January 3rd, 2022

DOI: https://doi.org/10.21203/rs.3.rs-1210143/v1

License: (9) This work is licensed under a Creative Commons Attribution 4.0 International License. Read Full License 


\section{A STOCHASTIC MODEL FOR KALA-AZAR TRANSMISSION DYNAMICS IN LIBO KEMKEM, ETHIOPIA}

Sewmehon Shimekaw Alemu

Physics, University of Gondar, Gondar, Ethiopia

Email: shisewmehon@gmail.com

Tel: $+251-910808023$

P.O.Box: 196, University of Gondar, Ethiopia

Mr. Sewmehon Shimekaw is a Lecturer in University of Gondar, College of Natural and Computational Sciences, Department of Physics, Gondar, Ethiopia. He holds a Master of Science in Statistical and Computational Physics (MSc. Statistical and Computational Physics) and a Bachelor of degree in Physics (BSc, Physics). He is engaged in teaching Physics courses at Undergraduate, Research Supervision in the said areas and levels, and community engagement, among others. His main areas of research are mathematical modelling of infectious diseases and mathematical and computational studies of biological systems.

\section{A STOCHASTIC MODEL FOR KALA-AZAR TRANSMISSION DYNAMICS IN LIBO KEMKEM, ETHIOPIA}

Abstract: The objective of this paper is to analyse and demonstrate the dynamics of Kala-azar infected group using stochastic model, particularly using simple SIR model with python script over time. The model is used under a closed population with $\mathbf{N}=\mathbf{1 0 0}$, transmission rate coefficient $\boldsymbol{\beta}=\mathbf{0 . 0 9}$, recovery rate $\boldsymbol{\gamma}=\mathbf{0 . 0 3}$ and initial condition $\mathbf{I}(\mathbf{0})=\mathbf{1}$. In the paper it is discussed how the Kala-azar infected group behaves through simple SIR model. The paper is completed with stochastic SIR model simulation result and shows stochasticity of the dynamics of Kala-azar infected population over time. Fig.2 below depicts continuous fluctuations which tells us the disease evolves with stochastic nature and shows random process.

Subject: Infectious Disease, Global Health, Health Informatics and Statistical and Computational Physics Key words: Stochastic Model, SIR Model, Infectious Disease, Kala-azar 


\section{Background of the Study}

Vector-borne diseases(VBD) are infections transmitted by the bite of infected arthropod(insect) species, such as mosquitoes, ticks, triatomine bugs, sand flies, and black flies[1]. Arthropod vectors are coldblooded (ectothermic) and thus especially sensitive to climatic factors. Weather influences survival and reproduction rates of vectors, in turn influencing habitat suitability, distribution and abundance; intensity andtemporal pattern of vector activity (particularly biting rates) throughout the year; and rates of development, survival and reproduction of pathogens within vectors[2].

"Kala-azar" (or Indian Visceral Leishmaniasis for "black fever") are a group of diseases with very complex dynamics caused by more than 20 species of the protozoan genus Leishmania that are transmitted between humans and other mammalian hosts by the vector phlebotomine sand-flies[3]. There are two major clinical forms of leishmaniasis, namely Cutaneous Leishmaniasis (CL) and Visceral Leishmaniasis (VL) depending on the species of leishmania responsible and the immune response to infection[4].

The cutaneous form tends to heal spontaneously leaving scars. Depending on the species of leishmania responsible it may evolve into diffuse cutaneous leishmaniasis, recidivate leishmaniasis, or mucocutaneous leishmaniasis, with disastrous aesthetic consequences for the patient.

Visceral leishmaniasis has two major forms leishmania donovani, and leishmania infantum. VL shows signs of fever, weight loss, splenomegaly, and anaemia and is the most severe form and fatal in almost all cases if left untreated. It may cause epidemic outbreaks with a high mortality rate. A varying proportion of visceral cases may evolve into a cutaneous form known as Post Kala-azar Dermal Leishmaniasis (PKDL), a nonfatal stage of infection dermatological symptoms, \& which requires lengthy and costly treatment[5].

The disease endangers some 350 million people in 98 countries, most of them in the poorer regions of the globe [6]. An estimated 200,000 to 400,000 new cases of VL occur worldwide each year. And from this, greater than $90 \%$ of VL human cases occur in six countries, namely Bangladesh, Brazil, Ethiopia, India, South Sudan and Sudan [7]. Eastern Africa has the second highest number of VL cases, after the Indian Subcontinent. The disease is endemic in Eritrea, Ethiopia, Kenya, Somalia, Sudan, South Sudan, and Uganda [8]. 
In Ethiopia, the first case of VL was documented in 1942 in the lower Omo plains, the southwestern part of the country [9]. The disease has spread to become endemic in many parts of the country. It is prevalent mostly in lowland, arid areas, and the parasite involved is mainly leishmania donovani, with an estimated annual incidence of more than 4,000 cases [10]. Most important endemic foci include the Humera and Metema plains in the northwest[11], the Omo plains, the Aba Roba focus, and the Weyto River Valley in the southwest of the country[12].

In the country, VL mainly occurs in the arid and semi-arid areas; however, recent reports indicate spreading of the disease to areas where it was previously non-endemic[13]. In 2003, an outbreak of VL occurred in highland areas of the Libo Kemkem district, in the Amhara regional state[14].

VL is becoming a growing public health threat; the spatial distribution and burden of VL is up surging year after year[15]. VL-HIV co-infection is rising in Ethiopia, and it poses a new and difficult challenge to VL control effort[16]. VL-HIV co-infection is characterized by a number of complexities, including challenging diagnosis, increased drug toxicity, and poor treatment response. Leishmaniasis in Ethiopia was formerly overseen by the Ministry of Health $(\mathrm{MoH})$, but after the $\mathrm{MoH}$ underwent a large reorganization in 2007, a national leishmaniasis task force was established with the aim of eliminating VL by 2015[17]. Efforts made by the MoH so far to control VL are not withstanding its upsurge and, hence, VL is developing both on a spatial and temporal basis. Considering its recent upsurging, I thoroughly reviewed and analysed the previous works done, and I also propose a way forward to tackle this disease.

\subsection{Statement of the problem}

Conducting epidemiology research that deals with the vector-borne disease has a long history[18]. Plenty of these studies used mathematical models. In this paper I focused on the analysis of the dynamics of Kala-azar disease using stochastic model. 


\section{Models and method}

In this paper the dynamics of Kala-azar disease is modelled using Stochastic SIR model. The model is developed with the data collected from Addis Zemen primary hospital report and it is simulated with python programming software.

\subsection{Model Formulation}

In the SIR epidemic model of[19], the population is divided into three categories of individuals: $\mathrm{S}$ the susceptible individuals, I the infected individuals and $\mathrm{R}$ the recovered and immune individuals. The parameters of the model are denoted by $\beta$ and $\gamma$.

- $\beta$ is the transmission rate coefficient of infections in a time period (with $\beta$ greater or equal to zero) and its value can be easily determined from the solutions of deterministic model.

- $\gamma$ is the recovery rate (with $\gamma$ greater or equal to zero).

A compartmental diagram in Fig.1 below illustrates the relationship between the three classes(S, I and R). Arrows denotes possible changes and transition probabilities.

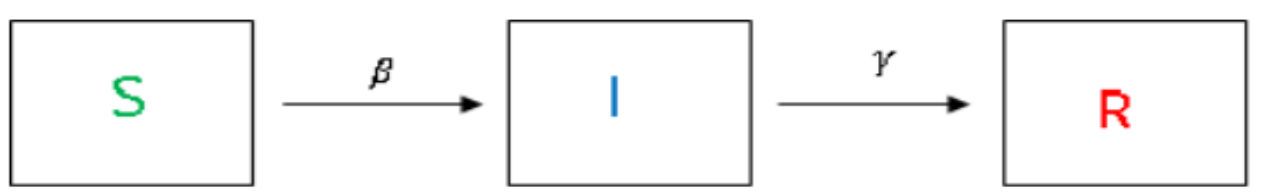

Figure 1: The SIR compartmental model diagram.

\subsubsection{Model Assumptions}

The SIR Model is used in epidemiology to compute the amount of susceptible, infected and recovered people in a population. This model is an appropriate one to use under the following assumptions[20]:

1. The population is fixed (100 populations in this case).

2. The only way a person can leave the susceptible group is to become infected. The only way a person can leave the infected group is to recover from the disease. Once a person has recovered, the person received immunity.

3. Age, sex, social status, and race do not affect the probability of being infected.

4. There is no inherited immunity. 
5. The member of the population mix homogeneously (have the same interactions with one another to the same degree).

\subsubsection{Equations of the model}

Assume the time variable is continuous, $t \in[0, \infty)$ and the states $S(t), I(t)$ and $R(t)$ are continuous random variables, that is,

$$
S(t), I(t), R(t) \in[0, N]
$$

The SIR epidemic process is bivariate, $\{\mathrm{S}(\mathrm{t}), \mathrm{I}(\mathrm{t})\}$, where $\mathrm{R}(\mathrm{t})=\mathrm{N}-\mathrm{S}(\mathrm{t})-\mathrm{I}(\mathrm{t})[21]$.

And where

$$
\begin{aligned}
& \mathrm{S}(\mathrm{t})=\text { is the number of susceptible individuals to VL at time } \mathrm{t} \text {. } \\
& \mathrm{I}(\mathrm{t})=\text { is the number of infected individuals with VL at time } \mathrm{t} \text {. } \\
& \mathrm{R}(\mathrm{t})=\text { is the number of recovered individuals from VL at time } \mathrm{t} \text {. } \\
& \text { and } \mathrm{N} \text { is the total population size. }
\end{aligned}
$$

The random variable $\mathrm{I}(\mathrm{t})$ has an associated probability density function $(\mathrm{pdf}), p(x, t)$,

$$
\operatorname{Prob}\{a \leq I(t) \leq b\}=\int_{a}^{b} p(x, t) d x
$$

For the stochastic SIR epidemic model, it can be shown that the pdf satisfies a forward Kolmogorov differential equation.

Assume $\operatorname{Prob}\left\{I(0)=i_{0}\right\}=1$ and

Let $p\left(x, t ; i_{0}, i\right)=p(i, t)=p_{i}(t)$

Then the system of differential equations for the CTMC model[22]

$$
\frac{d p_{i}}{d t}=p_{i-1} b(i-1)+p_{i+1} d(i-1)-p_{i}[b(i)+d(i)]
$$

For $i=1,2, \ldots, N \& \frac{d p_{0}}{d t}=p_{1} d(i)$ can be expressed as a finite difference scheme in the variable $i$ with $\Delta i=1$,

$$
\begin{aligned}
\frac{d p_{i}}{d t} & =p_{i-1} b(i-1)+p_{i+1} d(i-1)-p_{i}[b(i)+d(i)] \\
& =-\frac{\left\{p_{i+1}[b(i+1)-d(i+1)]-p_{i-1}[b(i-1)-d(i-1)]\right\}}{2 \Delta i}
\end{aligned}
$$




$$
+\frac{1}{2} \frac{\left\{p_{i+1}[b(i+1)+d(i+1)]-2 p_{i}[b(i)+d(i)]+p_{i-1}[b(i-1)+d(i-1)]\right\}}{(\Delta i)^{2}}
$$

Let $i=x, \Delta i=\Delta x$ and $p_{i}(t)=p(x, t)$. Then the limiting form of the preceding equation (as $\left.\Delta x \rightarrow 0\right)$ is the forward Kolmogorov differential equation for $p(x, t)$ :

$$
\frac{\partial p(x, t)}{\partial t}=-\frac{\partial}{\partial x}\{[b(x)-d(x)] p(x, t)\}+\frac{1}{2} \frac{\partial^{2}}{\partial x^{2}}\{[b(x)+d(x)] p(x, t)\}
$$

Substituting $b(x)=\beta x(N-x) / N$ and $d(x)=(b+\gamma) x$ yields

$$
\begin{gathered}
\frac{\partial p(x, t)}{\partial t}=-\frac{\partial}{\partial x}\left\{\left[\frac{\beta}{N} x(N-x)-(b+\gamma) x\right] p(x, t)\right\} \\
+\frac{1}{2} \frac{\partial^{2}}{\partial x^{2}}\left\{\left[\frac{\beta}{N} x(N-x)+(b+\gamma) x\right] p(x, t)\right\}
\end{gathered}
$$

The coefficient of $p(x, t)$ in the first term on the right side of the preceding equation, $[\beta x(N-x) / N-(b+\gamma) x]$ is the infinitesimal mean and the coefficient of $p(x, t)$ in the second term, $[\beta x(N-x) / N+(b+\gamma) x]$ is the infinitesimal variance.

In the stochastic model, $\boldsymbol{S}=(S, I)^{T}$ is composed of continuous random variables. Now, consider the events that may occur independently within a small time interval $\Delta t$ while neglecting the possibility of multiple events of order $(\Delta t)^{2}$. The possible changes in the two populations ( $S$ and $I$ ) that append and their corresponding probabilities are shown in the Table 1 below.

\begin{tabular}{|c|c|l|}
\hline Change & Probability & Event \\
\hline$\Delta \boldsymbol{S}^{(1)}=(-1,1)^{T}$ & $p_{1}=\beta \frac{S I}{N} \Delta t$ & $S$ Infected \\
\hline$\Delta \boldsymbol{S}^{(2)}=(0,-1)^{T}$ & $p_{2}=\gamma I \Delta t$ & $I$ Recovered \\
\hline
\end{tabular}

Table 1: Changes and corresponding probabilities in the SIR model.

where $\boldsymbol{S}$ denotes the state (S or $I$ )

The vector $\Delta \boldsymbol{S}^{(1)}=(-1,1)^{T}$ represents the change of one individual from population $S$ in to $I$ (the infection of one individual) during $\Delta t$ with a probability that is proportional to $S[23]$. And the vector $\Delta \boldsymbol{S}^{(2)}=(0,-1)^{T}$ represents a recovery of a population $I$ with probability $p_{2}=\gamma I \Delta t$. 
We deduce that the dynamics of the two interacting populations is an Itô stochastic differential equation (SDE) of the form

$$
d S(t)=\mu(t, S, I) d t+B(t, S, I) d W(t)
$$

with $S(0)=S_{0}$, where $W(t)=\left(W_{1}(t), W_{2}(t)\right)^{T}$ is a two-dimensional winner process.

Let $\Delta S(t)=(\Delta S, \Delta I)^{T}$, then the expectation vector $\mu$ and the variance covariance matrix $\boldsymbol{V}$ of $\Delta S(t)$ to order $\Delta t$ are

$$
\begin{aligned}
\mu & =E[\Delta S] / \Delta t \\
& =\left(\begin{array}{c}
-\frac{\beta}{N} S I \\
\frac{\beta}{N} S I-\gamma I
\end{array}\right),
\end{aligned}
$$

Where,

$$
\begin{aligned}
E[\Delta S] & =\sum_{i=1}^{2} p_{i} \Delta S^{i} \\
& =\left(\begin{array}{c}
-\frac{\beta}{N} S I \\
\frac{\beta}{N} S I-\gamma I
\end{array}\right) \Delta t \\
V & =E\left[\Delta S . \Delta S^{T}\right] / \Delta t \\
& =\left(\begin{array}{cc}
\frac{\beta}{N} S I & -\frac{\beta}{N} S I \\
-\frac{\beta}{N} S I & \frac{\beta}{N} S I+\gamma I
\end{array}\right) \\
& =\frac{\beta}{N} S I\left(\begin{array}{cc}
1 & -1 \\
-1 & 1+\frac{\gamma N}{\beta S}
\end{array}\right),
\end{aligned}
$$

where

$$
\begin{aligned}
E\left[\Delta S . \Delta S^{T}\right] & =\sum_{i=1}^{2} p_{i} \Delta S^{i} . \Delta S^{(i) T} \\
& =\left(\begin{array}{cc}
\delta & -\delta \\
-\delta & \gamma I+\delta
\end{array}\right) \\
& =\left(\begin{array}{cc}
\frac{\beta}{N} S I & -\frac{\beta}{N} S I \\
-\frac{\beta}{N} S I & \frac{\beta}{N} S I+\gamma I
\end{array}\right) \Delta t
\end{aligned}
$$

where $\quad \delta=\frac{\beta}{N} S I$

using the Allen's formula[24], [25],

$$
V=\left(\begin{array}{ll}
V_{11} & V_{12} \\
V_{21} & V_{22}
\end{array}\right)
$$


the root squared matrix $B=\mathbf{V}^{1 / 2}$ become

$$
\begin{aligned}
\mathbf{B} & =\mathbf{V}^{1 / 2} \\
& =\frac{1}{d}\left(\begin{array}{cc}
V_{11}+\delta & V_{12} \\
V_{21} & V_{22}+\delta
\end{array}\right),
\end{aligned}
$$

where $d=\sqrt{\operatorname{tr}(\mathbf{V})+2 \delta}$ and $\delta=\sqrt{\operatorname{det}(\mathbf{V})}$

$$
\mathbf{B}=\frac{\sqrt{\beta S I / N}}{\sqrt{2+\frac{\gamma N}{\beta S}+2 \sqrt{\frac{\gamma N}{\beta S}}}}\left(\begin{array}{cc}
1+\sqrt{\frac{\gamma N}{\beta S}} & -1 \\
-1 & 1+\frac{\gamma N}{\beta S}+\sqrt{\frac{\gamma N}{\beta S}}
\end{array}\right)
$$

The expectation vector $\mu(t, S, I)=E[\Delta S] / \Delta t$ and the root square matrix $\mathrm{B}=\mathrm{V}^{1 / 2}$ respectively expresses the drift and the diffusion coefficients of a stochastic process. Omitting the time argument, the SDE of the stochastic SIR model in matrix form is

$$
\left(\begin{array}{l}
d S \\
d I
\end{array}\right)=\left(\begin{array}{c}
-\frac{\beta}{N} S I \\
\frac{\beta}{N} S I-\gamma I
\end{array}\right) d t+\frac{\sqrt{\beta S I / N}}{\sqrt{2+\frac{\gamma N}{\beta S}+2 \sqrt{\frac{\gamma N}{\beta S}}}}\left(\begin{array}{cc}
1+\sqrt{\frac{\gamma N}{\beta S}} & -1 \\
-1 & 1+\frac{\gamma N}{\beta S}+\sqrt{\frac{\gamma N}{\beta S}}
\end{array}\right)\left(\begin{array}{l}
d W_{1} \\
d W_{2}
\end{array}\right)
$$

The drift and diffusion terms determine the change in number of infections over time[26].

The ratio $\beta S / N$ is the proportion of contacts by one infected individual and $(\beta S / N) I$ the number of contacts by the infected population. The ratio $1 / \gamma$ denotes the average length of the infection period[27].

As [28] by applying Euler's method of systems, we can solve the deterministic model

$$
\left\{\begin{array}{l}
\frac{d S(t)}{d t}=-\frac{\beta I(t)}{N} S(t) \\
\frac{d I(t)}{d t}=\frac{\beta S(t)}{N} I(t)-\gamma I(t) \\
\frac{d S(t)}{d t}=\gamma I(t)
\end{array}\right.
$$

From these equations, we can discover how the different groups will act as $t \rightarrow \infty$.

From equation (12) of the first term, we understand that the susceptible group will decrease over time and approach zero. From the third term of equation (12), we also understand that the recovered group increase and will approach $N$ over time. However, in the second term of the equation it is difficult to understand easily how the infected group behaves. 
The solutions to the deterministic differential equations are:

$$
\begin{aligned}
S_{n+1} & =S_{n}-\beta S_{n} I_{n} \Delta t \\
I_{n+1} & =I_{n}\left(1+\left(\beta S_{n}-\gamma\right) \Delta t\right) \\
R_{n+1} & =R_{n}+\gamma I_{n} \Delta t
\end{aligned}
$$

where $S_{n+1}, I_{n+1}$ and $R_{n+1}$ are the number of susceptible, infected and recovered people at time $(n+1) . \Delta t$ is a small change in time, and will be equal to one.

In SIR model assumptions, birth rate and death is zero $(b=d=0)$. A key threshold outcome of an epidemic model is typically determined by the basic reproductive number, often denoted by $\mathrm{R}_{0}$. It is defined as the total number of secondary infections caused by single infected individuals. This threshold property provides important information about the potential of disease and impact of control mechanism. An epidemic will outbreak if and only if this number is greater than one else the disease is die out. The basic reproduction number is defined[29] as follows:

$$
R_{0}=\beta /(b+\gamma)
$$

From equation $(16)$, the fraction $1 /(b+\gamma)$ is the length of the infectious period, adjusted for deaths. The fraction gamma $(\gamma)$ of infected recovering in a given day can be estimated from observation of infected individuals. Specifically, $\gamma$ is roughly the reciprocal of the number of days an individual is sick enough to infect others.

If $\mathrm{R}_{0}<1$, each individual produces on average less than one new infected individual and hence the disease dies out and if $\mathrm{R}_{0}>1$, each individual produces more than one new infected individual and hence the disease is able to invade the susceptible population[30]. The quantity $\mathrm{R}_{0} \frac{\mathrm{S}_{(0)}}{\mathrm{N}}$ is referred to as the initial replacement number the average number of secondary infections produced by an infected individual during the period of infectiousness at the outset of the epidemic. Since the infectious fraction changes during the course of the epidemic, the replacement number is generally defined as $\mathrm{R}_{0} \frac{\mathrm{S}_{(0)}}{\mathrm{N}}$. If $\mathrm{R}_{0} \frac{\mathrm{S}_{(0)}}{\mathrm{N}}>1$, then there is an initial increase in the number of infected cases $I_{(t)}$ (epidemic) and the population experiences an outbreak, but if $R_{0} \frac{S_{(0)}}{N} \leq 1$, 
then $I_{(t)}$ decreases monotonically to zero (disease-free equilibrium) and the disease eventually disappears from the population.

The probability of an outbreak is $\approx\left\{\begin{array}{cc}0 & \text { if } R_{0} \leq 1 \\ 1-\left(\frac{1}{R_{0}}\right)^{i_{0}} & \text { if } R_{0}>1\end{array}\right.$

\subsubsection{Stochastic Simulation Algorithm}

A version of the stoch_eqs algorithm is used to code this model as detailed in the procedure below:

Step 1. Initialize the infected population of the system $I_{0}=1$;

Step 2. Calculate the transition rates for the given population $I$ of the system;

Step 3. Simulate the time, until the next transition by drawing from an exponential distribution;

Step 4. Simulate the transition type by drawing from the discrete distribution. Generate a random number $I$ from a uniform distribution and choose the transition as follows:

If $I_{1}<\left(\right.$ Rate $_{1} /\left(\right.$ Rate $_{1}+$ Rate $\left.\left._{2}\right)\right)$ :

choose transition 1 (do infection

else:

choose transition 2 (do recovery);

Step 5. Update the new time and the new system population;

Step 6. Iterate steps $2-5$ until $t \geq t_{\text {stop }}$

\section{Result and discussion}

In this work, stochastic model is formulated and analysed for SIR model with a closed population made up of 100 people that mix homogeneously. Since the disease is highly infectious, everyone will eventually become infected. From the population how many people will be infected by the disease in each state at a given period of time which is collected from Addis Zemen primary hospital report(in Libo Kemkem district) shows the infected population increases at the middle months of the year and it is shown in Table 2 below. This is because of the sand fly highly incubated in those months in the study area. At the beginning, 99 persons were 
susceptible to the disease, and the one had been infected by the disease. A period also lasts 29 days, an estimated average duration of Kalazar infection obtained from the report. In these cases, everyone recover in one period of 29 days (the average period of infectiousness at). From this we have the recovery rate $\gamma=1 / 29=0.03$

\begin{tabular}{|c|c|c|c|c|c|c|c|c|c|c|c|c|c|}
\hline & Period & 0 & 1 & 2 & 3 & 4 & 5 & 6 & 7 & 8 & 9 & 10 & 11 \\
\hline \multirow{3}{*}{ 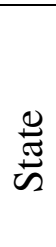 } & $\mathrm{S}$ & 99 & 98 & 95 & 82 & 67 & 33 & 16 & 7 & 3 & 1 & 0 & 0 \\
\hline & $\mathrm{I}$ & 1 & 1 & 3 & 13 & 15 & 34 & 17 & 9 & 4 & 2 & 1 & 0 \\
\hline & $\mathrm{R}$ & 0 & 1 & 2 & 5 & 18 & 33 & 67 & 84 & 93 & 97 & 99 & 100 \\
\hline
\end{tabular}

Table 2: The number of cases in each state per period.

From Tables 2, we can get $\beta$. To do this we can use equation (13), with $\Delta t=1$ to get the following

$$
\beta=\frac{S_{n}-S_{n+1}}{S_{n} I_{n}}
$$

Using this equation we can get $\beta$ for each period. The $\beta$ values are calculated for each period and the average value is obtained to 0.09 as shown in the Table 3 below.

\begin{tabular}{|c|l|l|l|l|l|l|l|l|l|l|l|l|l|}
\hline Period & 0 & 1 & 2 & 3 & 4 & 5 & 6 & 7 & 8 & 9 & 10 & 11 & Average \\
\hline$\beta$ & 0.0 & 0.01 & 0.03 & 0.05 & 0.01 & 0.03 & 0.02 & 0.03 & 0.06 & 0.30 & 0.50 & 0.00 & 0.09 \\
\hline
\end{tabular}

Table 3: The different $\beta^{\prime}$ s for each period and the average $\beta$.

The number of infected population - time graph is depicted in Fig.2 by one sample path of the SDE SIR model below. The parameter values are, $\Delta t=1, \beta=0.09, \gamma=0.03$ and $N=100$ with initial condition $I(0)=1$. 


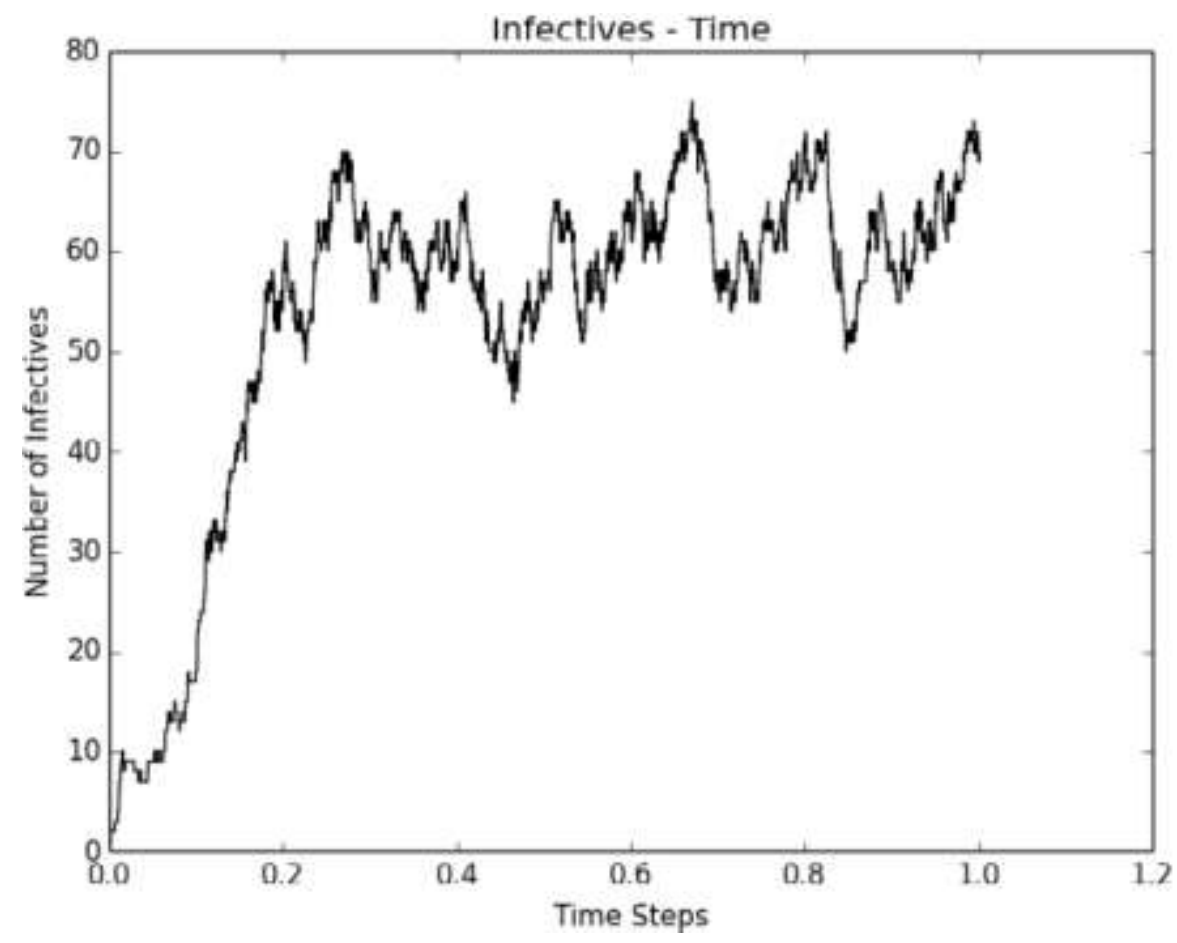

Figure 2: SDE SIR model of Kala-azar disease with parameter values:

$$
\Delta t=1, \beta=0.09, \gamma=0.03 \text { and } N=100 \text { with initial condition } I(0)=1 .
$$

The SDE SIR Model python simulation result plotted in fig.2, under a closed population with $N=100$, transmission rate coefficient $\beta=0.09$, recovery rate $\gamma=0.03$ and initial condition $I(0)=1$; the dynamics of Kala-azar infected populations over time fluctuates continuously and evolves with stochastic behavior. This shows us that the dynamical behavior of the disease has random nature.

Fig.3 below shows the Wiener process has a stationary independent increment that is the increments $\Delta \mathrm{W}$ depend only on $\Delta \mathrm{t}$. They are independent of $\mathrm{t}$. The notation $\frac{\mathrm{dW}}{\mathrm{dt}}$ is only for convenience because sample paths of $\mathrm{W}(\mathrm{t})$ are continuous but not where differentiable. 


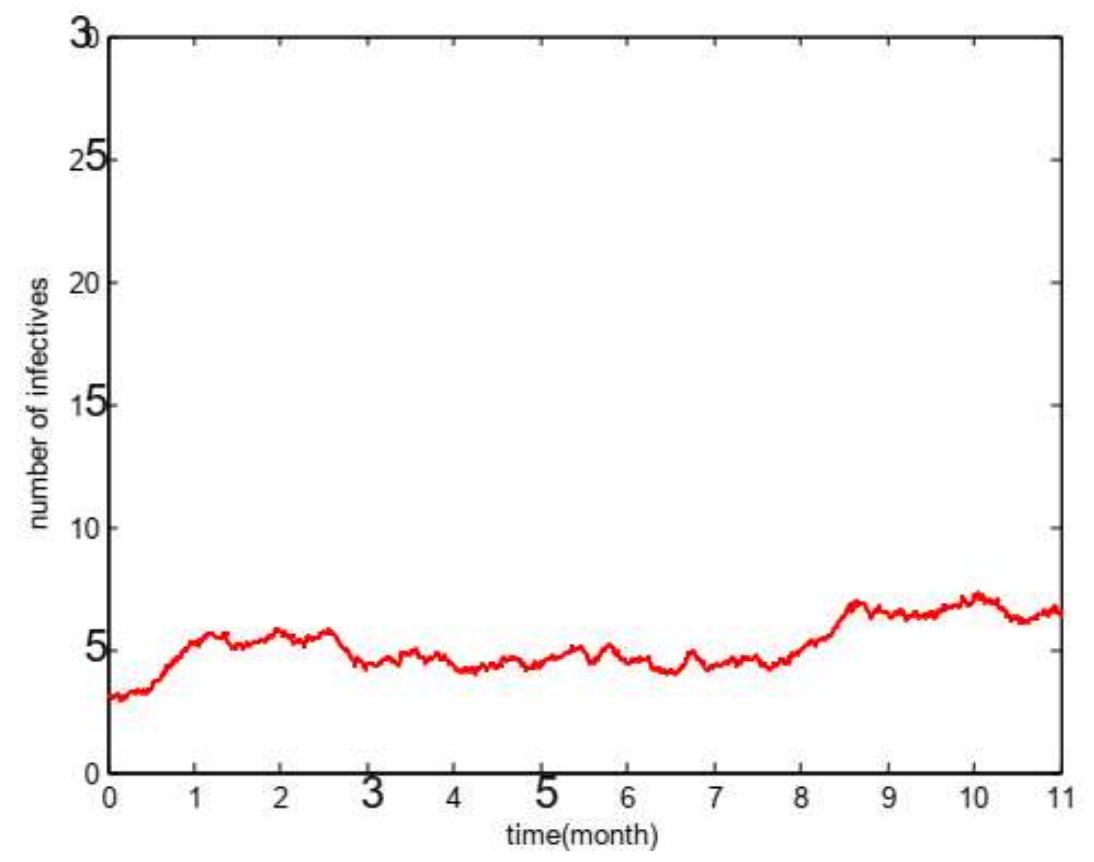

Figure 3: Sample Paths of a Wiener Process.

\section{Conclusion}

It can be concluded SDE SIR Model is used in the modelling of infectious diseases by computing the amount of people in a closed population that are susceptible, infected, or recovered at a given period of time. In this work a stochastic SIR model without the presence of birth and death rate in the population is applied to analyse the transmission of Kala-azar disease. I have shown the stochasticity (random nature) of the dynamics of Kala-azar infected population using SIR model. The disease is endemic in the study area $\left(\mathrm{R}_{0}>1\right)$. Reproductive number, can be reduced through an increase in the recovery rate through medication[31].

\section{Abbreviations}

CL: Cutaneous Leishmaniasis

CTMC: Continuous Time Markov Chain

MoH: Ministry of Health

pdf: probability density function

PKDL: Post Kala-azar Dermal Leishmaniasis

SDE: Stochastic Differential Equation 
SIR: Susceptible - Infected - Recovered

VBD: Vector-borne diseases

VL: Visceral Leishmaniasis

VL-HIV: Visceral Leishmaniasis - Human Immune Virus

\section{Acknowledgements}

The author would like to thank the individuals working in Addis Zemen Primary Hospital, responsible for data collection.

\section{Funding}

The author received no direct funding for this research.

\section{Author details}

Sewmehon Shimekaw Alemu

E-mail: shisewmehon@gmail.com

Department of Physics, University of Gondar, College of Natural and Computational Sciences, Gondar, Ethiopia.

\section{Availability of data and materials}

The data sets analysed during the current study are available from the corresponding author on reasonable request.

\section{Disclosure of potential conflicts of interest}

The author has declared that he has no competing interests.

\section{Ethical considerations}

All procedures performed in this study were in accordance with the ethical standards of University of Gondar Institution and the University Research Committee declarations with comparable ethical standards for confidentiality. 


\section{References}

[1] R. S. K. Uk, “IPCC: Ch 8. Human Health,” Change, pp. 391-431, 2007.

[2] D. J. Rogers and S. E. Randolph, “Climate Change and Vector-Borne Diseases,” Adv. Parasitol., vol. 62, no. 05, pp. 345-381, 2006, doi: 10.1016/S0065-308X(05)62010-6.

[3] D. E. A. Elnaiem, H. K. Hassan, O. F. Osman, R. D. C. Maingon, R. Killick-Kendrick, and R. D. Ward, "A possible role for Phlebotomus (Anaphlebotomus) rodhaini (Parrot, 1930) in transmission of Leishmania donovani," Parasites and Vectors, vol. 4, no. 1, pp. 2-7, 2011, doi: 10.1186/1756-3305$4-238$.

[4] A. Moncaz, R. Faiman, O. Kirstein, and A. Warburg, "Breeding sites of Phlebotomus sergenti, the sand fly vector of cutaneous leishmaniasis in the Judean desert," PLoS Negl. Trop. Dis., vol. 6, no. 7, 2012, doi: 10.1371/journal.pntd.0001725.

[5] “Control of the leishmaniases: Report of a WHO expert committee," World Health Organization Technical Report Series, no. 793. pp. 9-131, 1990.

[6] M. Worku Lake, "Epidemiological Analysis of Malaria Outbreak in Ankesha District, Awi Zone, Amhara Region, Ethiopia, 2012: Weaknesses in Control Measures and Risk Factors," Sci. J. Public Heal., vol. 4, no. 2, p. 132, 2016, doi: 10.11648/j.sjph.20160402.18.

[7] S. Sato, "Correction to: Plasmodium — a brief introduction to the parasites causing human malaria and their basic biology (Journal of Physiological Anthropology, (2021), 40, 1, (1), 10.1186/s40101-02000251-9),” J. Physiol. Anthropol., vol. 40, no. 1, pp. 1-13, 2021, doi: 10.1186/s40101-021-00254-0.

[8] R. Rosenberg, "Plasmodium vivax in Africa: hidden in plain sight?," Trends Parasitol., vol. 23, no. 5, pp. 193-196, 2007, doi: 10.1016/j.pt.2007.02.009.

[9] R. E. Howes et al., "Global epidemiology of Plasmodium vivax," Am. J. Trop. Med. Hyg., vol. 95, no. Suppl 6, pp. 15-34, 2016, doi: 10.4269/ajtmh.16-0141.

[10] G. D. Shanks and N. J. White, "The activation of vivax malaria hypnozoites by infectious diseases," Lancet Infect. Dis., vol. 13, no. 10, pp. 900-906, 2013, doi: 10.1016/S1473-3099(13)70095-1.

[11] G. P. Garnett, S. Cousens, T. B. Hallett, R. Steketee, and N. Walker, "Mathematical models in the 
evaluation of health programmes," Lancet, vol. 378, no. 9790, pp. 515-525, 2011, doi: 10.1016/S0140-6736(10)61505-X.

[12] F. Bekele, T. Belay, A. Zeynudin, and A. Hailu, "Visceral leishmaniasis in selected communities of Hamar and Banna-Tsamai districts in Lower Omo Valley, South West Ethiopia: Sero-epidemological and Leishmanin Skin Test Surveys," PLoS One, vol. 13, no. 5, pp. 1-13, 2018, doi: 10.1371/journal.pone.0197430.

[13] D. Gebremichael, "Zoonotic impact and epidemiological changes of leishmaniasis in Ethiopia," Open Vet. J., vol. 8, no. 4, pp. 432-440, 2018, doi: 10.4314/ovj.v8i4.13.

[14] J. Alvar et al., "Kala-Azar outbreak in Libo Kemkem, Ethiopia: Epidemiologic and parasitologic assessment,” Am. J. Trop. Med. Hyg., vol. 77, no. 2, pp. 275-282, 2007, doi: 10.4269/ajtmh.2007.77.275.

[15] The World Health, "World Health Report Life in the 21st century A vision for all Report of the Director-General," 51st World Heal. Assem., 1998.

[16] E. Diro, L. Lynen, K. Ritmeijer, M. Boelaert, A. Hailu, and J. van Griensven, "Visceral Leishmaniasis and HIV Coinfection in East Africa," PLoS Negl. Trop. Dis., vol. 8, no. 6, 2014, doi: 10.1371/journal.pntd.0002869.

[17] S. Leta, T. H. T. Dao, F. Mesele, and G. Alemayehu, "Visceral Leishmaniasis in Ethiopia: An Evolving Disease,” PLoS Negl. Trop. Dis., vol. 8, no. 9, 2014, doi: 10.1371/journal.pntd.0003131.

[18] P. Nielsen, “Coastal and estuarine processes," Coastal And Estuarine Processes. pp. 1-360, 2009, doi: $10.1142 / 7114$.

[19] O. N. Bjørnstad, K. Shea, M. Krzywinski, and N. Altman, "The SEIRS model for infectious disease dynamics," Nat. Methods, vol. 17, no. 6, pp. 557-558, 2020, doi: 10.1038/s41592-020-0856-2.

[20] D. Smith, L. Moore, J. C. Chuang, and J. Michel, “The SIR model for spread of disease,” J. Online Math. its Appl., vol. 1, no. 3, pp. 1-5, 2001.

[21] P. Van Den Driessche, Deterministic compartmental models: Extensions of basic models, vol. 1945. 2008. 
[22] A. J. Ekanayake and L. J. S. ALLEN, “Comparison of markov chain and stochastic differential equation population models under higher-order moment closure approximations," Stoch. Anal. Appl., vol. 28, no. 6, pp. 907-927, 2010, doi: 10.1080/07362990903415882.

[23] L. J. S. Allen, “A primer on stochastic epidemic models: Formulation, numerical simulation, and analysis," Infect. Dis. Model., vol. 2, no. 2, pp. 128-142, 2017, doi: 10.1016/j.idm.2017.03.001.

[24] A. A. Keller, "Population biology models with time-delay in a noisy environment," WSEAS Trans. Biol. Biomed., vol. 8, no. 4, pp. 113-134, 2011.

[25] E. J. Allen, "Derivation and computation of discrete-delay and continuous-delay sdes in mathematical biology," Math. Biosci. Eng., vol. 11, no. 3, pp. 403-425, 2014, doi: 10.3934/mbe.2014.11.403.

[26] L. J. S. Allen, “An introduction to stochastic epidemic models,” Lect. Notes Math., vol. 1945, pp. 81130, 2008, doi: 10.1007/978-3-540-78911-6_3.

[27] Y. Song, T. Zhang, H. Li, K. Wang, and X. Lu, "Mathematical Model Analysis and Simulation of Visceral Leishmaniasis, Kashgar, Xinjiang, 2004-2016,” Complexity, vol. 2020, 2020, doi: $10.1155 / 2020 / 5049825$.

[28] J. P. Maassen, “The SIR and SEIR Epidemiological Models Revisited," Preprints, no. May, pp. 1-14, 2020, doi: 10.20944/preprints202005.0090.v1.

[29] K. Dietz, "The estimation of the basic reproduction number for infectious diseases," Stat. Methods Med. Res., vol. 2, no. 1, pp. 23-41, 1993, doi: 10.1177/096228029300200103.

[30] O. Diekmann, J. A. P. Heesterbeek, and J. A. J. Metz, "On the definition and the computation of the basic reproduction ratio R0 in models for infectious diseases in heterogeneous populations," J. Math. Biol., vol. 28, no. 4, pp. 365-382, 1990, doi: 10.1007/BF00178324.

[31] N. Chitnis, "Einf" uhrung in die Mathematische Epidemiologie : Introduction to Mathematical Epidemiology : Deterministic Compartmental Models,”pp. 1-12, 2011. 


\section{Supplementary Files}

This is a list of supplementary files associated with this preprint. Click to download.

- GraphycalAbstract.docx 